\title{
Certificate License Text
}

National Cancer Institute

\section{Source}

National Cancer Institute. Certificate License Text. NCI Thesaurus. Code C93527.

A character string that describes the certification or specific licensed credentials of the qualified entity. 